Check for updates

Cite this: J. Mater. Chem. C, 2021, 9, 10741

Received 18th March 2021,

Accepted 23rd April 2021

DOI: $10.1039 / \mathrm{d} 1 \mathrm{tc} 01261 \mathrm{~d}$

rsc.li/materials-c

\section{Statics and dynamics of ferroelectric domains in molecular multiaxial ferroelectric $\left(\mathrm{Me}_{3} \mathrm{NOH}\right)_{2}\left[\mathrm{KCO}(\mathrm{CN})_{6}\right] \dagger$}

\author{
Wei-Jian Xu, ${ }^{\star a b c}$ Konstantin Romanyuk, ${ }^{\text {cd }}$ Ying Zeng, ${ }^{a}$ Andrei Ushakov, (D) ${ }^{d}$ \\ Vladimir Shur, ${ }^{d}$ Alexander Tselev, (D ' Wei-Xiong Zhang, iD *a Xiao-Ming Chen, ${ }^{a}$ \\ Andrei Kholkin (iD *cd and João Rocha (iD *b
}

\begin{abstract}
The recent emergence of multiaxial molecular ferroelectrics opens up a new route toward technological evolution in the next-generation flexible/wearable device applications. However, a fundamental understanding of multiaxial ferroelectricity and polarization switching at the microscopic level in these materials is still missing. Herein, we study a high-temperature multiaxial perovskite ferroelectric $\left(\mathrm{Me}_{3} \mathrm{NOH}\right)_{2}\left[\mathrm{KCO}(\mathrm{CN})_{6}\right](\mathrm{TMC}-4)$ that exhibits a bond-switching phase transition at $417 \mathrm{~K}$ with notable piezoelectricity and spontaneous polarization in the ferroelectric phase. The cleavage and reformation of coordination bonds and hydrogen bonds during the bond-switching transition all contribute to a large entropy change of $178.79 \mathrm{~J} \mathrm{~K}^{-1} \mathrm{~kg}^{-1}$ at the phase transition. Using piezoresponse force microscopy (PFM), we observed diverse ferroelectric domain structures and provide evidence for both $180^{\circ}$ and non- $180^{\circ}$ domain switching and their possible effect on the functional properties of molecular ferroelectrics. The results provide an insight into the multiaxial ferroelectricity of TMC-4 at the microscopic level enabling its further use in device applications.
\end{abstract}

\section{Introduction}

Molecular ferroelectrics have attracted significant attention due to their outstanding ferroelectricity-related properties comparable to those of pure inorganic oxides, ${ }^{1-7}$ structural flexibility, and tunability. ${ }^{8-11}$ Early studies have shown that molecular ferroelectrics are uniaxial, ${ }^{12-19}$ so that their ferroelectric functionality was restricted in a single-crystal form because the polarization could only be switched along the single polarization axis. With the development of targeted design strategies, ${ }^{20-23}$ multiaxial ferroelectricity was further discovered in molecular systems, such as hybrid perovskites, ${ }^{24-26}$ simple salts, ${ }^{27-32}$ and plastic crystals, ${ }^{33-35}$ in which several ferroelectric axes allow for arbitrarily directed

\footnotetext{
${ }^{a}$ MOE Key Laboratory of Bioinorganic and Synthetic Chemistry,

School of Chemistry, Sun Yat-Sen University, Guangzhou 510275, China. E-mail: zhangwx6@mail.sysu.edu.cn

${ }^{b}$ Department of Chemistry \& CICECO-Aveiro Institute of Materials, University of Aveiro,3810-193 Aveiro, Portugal. E-mail: rocha@ua.pt,weijxu@ua.pt ${ }^{c}$ Department of Physics \& CICECO-Aveiro Institute of Materials, University of Aveiro, 3810-193 Aveiro, Portugal. E-mail: kholkin@ua.pt

${ }^{d}$ School of Natural Sciences and Mathematics, Ural Federal University, 620000 Ekaterinburg, Russia

$\dagger$ Electronic supplementary information (ESI) available: Single-crystal structures, thermogravimetric analysis, and powder X-ray diffraction patterns. CCDC 2069810 and 2069811. For ESI and crystallographic data in CIF or other electronic format see DOI: 10.1039/d1tc01261d
}

polarization to orient along the electric field, hence rendering ferroelectric properties in a polycrystalline form. Moreover, due to several advantages such as light weight, mechanical flexibility, low acoustical impedance, and easy processability, the multiaxial molecular ferroelectrics are expected to replace the widely used inorganic oxides in the next-generation flexible electronics. ${ }^{36}$ Remarkably, great progress has recently been achieved in the field of multiaxial molecular ferroelectrics, including the discovery of the metal-free three-dimensional perovskite ( $N$-methyl-triethylenediaminium) $\mathrm{NH}_{4} \mathrm{I}_{3}$ exhibiting a large spontaneous polarization (up to $\left.22 \mu \mathrm{C} \mathrm{cm}{ }^{-2}\right),{ }^{37}$ ferroelectric $\mathrm{C}_{6} \mathrm{H}_{5}\left(\mathrm{CH}_{3}\right)_{3} \mathrm{CdCl}_{3}$ with a large shear strain of up to $21.5 \%,{ }^{2}$ and $\left(\mathrm{Me}_{3} \mathrm{NCH}_{2} \mathrm{~F}\right)_{x}\left(\mathrm{Me}_{3} \mathrm{NCH}_{2} \mathrm{Cl}\right)_{1-x} \mathrm{CdCl}_{3}$ $(x \approx 0.26)$ solid solution with giant piezoelectric coefficient $\left(d_{33}\right.$ of $\left.1540 \mathrm{pC} \mathrm{N}^{-1}\right) .{ }^{38}$

As a newcomer to the field of molecular ferroelectrics, multiaxial ferroelectricity may bring about much improved performance, relative to uniaxial materials. For instance, large

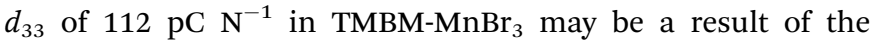
existence of multiple ferroelectric states induced by the significant symmetry change occurring at the paraelectric-to-ferroelectric phase transition. ${ }^{39}$ Therefore, they typically possess highly switchable polarization, remarkable piezoelectric effect due to the contribution of non $-180^{\circ}$ domain walls, and high pyroelectric performance. ${ }^{40,41}$ In this context, understanding ferroelectric domain structures in multiaxial ferroelectrics is essential to 
uncover the relationship between their macroscopic performance and microscopic polarization arrangements. With the advent of high-spatial-resolution imaging techniques, piezoresponse force microscopy (PFM) has emerged as an extremely useful tool for imaging ferroelectric domain structures at the nanometer scale. ${ }^{42-45}$ PFM allows a comprehensive understanding of polarization ordering and switching at the microscopic level, offering an opportunity to discover new physical mechanisms. For instance, intriguing vortex domain structures were recently observed in a few multiaxial molecular ferroelectrics, ${ }^{46-48}$ similar to what was observed in inorganic ferroelectrics in the past decade.

In 2017, our group demonstrated a remarkable bond-switching phase transition in a molecular perovskite multiaxial ferroelectric, $\left(\mathrm{Me}_{3} \mathrm{NOH}\right)_{2}\left[\mathrm{KFe}(\mathrm{CN})_{6}\right]$ (TMC-1), with a high Curie temperature of $402 \mathrm{~K}$ and 24 equivalent polarization directions. ${ }^{49}$ Very recently, Rok et al., reported its Co analogue, ${ }^{50}$ i.e., $\left(\mathrm{Me}_{3} \mathrm{NOH}\right)_{2}\left[\mathrm{KCO}(\mathrm{CN})_{6}\right]$ (abbreviated here as TMC-4) that exhibits an improved thermal stability and an enhanced nonlinear optical performance over TMC-1, in accordance with our early observations. ${ }^{51}$ However, for such a novel multiaxial ferroelectric, a detailed study of its ferroelectric domain structure and electromechanical properties is still missing, i.e., the mechanism of polarization switching in TMC-4 is far from being understood. Herein, we systematically studied the TMC-4 ferroelectric polarization, via structural, thermal, optical, electrical, and PFM measurements. Using PFM, we have investigated the static domain structure and domain kinetics in TMC-4 crystals, aiming at understanding the mechanisms of multiaxial polarization rotation and their effect on domain reversal. The present results give an insight into the ferroelectric properties of TMC-4 at the microscopic level, prompting their further application in various devices.

\section{Experimental section}

\section{Synthesis}

All chemicals were commercially available and used without further purification. Diluted hydrochloric acid $(20 \mathrm{mmol})$ was added dropwise to an aqueous solution of trimethylamine $\mathrm{N}$-oxide dihydrate $(20 \mathrm{mmol})$. The solution was then added to an aqueous solution of $\mathrm{K}_{3} \mathrm{Co}(\mathrm{CN})_{6}(10 \mathrm{mmol})$. Pale-yellow block crystals of TMC-4 were obtained on slow evaporation after a few days. Yield: $85 \%$ based on $\mathrm{K}_{3}\left[\mathrm{Co}(\mathrm{CN})_{6}\right]$. Elemental analysis: calculated (\%) for TMC-4 $\left(\mathrm{C}_{12} \mathrm{H}_{20} \mathrm{~N}_{8} \mathrm{O}_{2} \mathrm{CoK}\right)$ : C, 35.47; N, 27.57; H, 4.96. Found, C, 35.55; N, 27.39; H, 4.86.

\section{X-Ray crystallography}

The in situ variable-temperature single-crystal X-ray diffraction intensity data were collected on a Rigaku XtaLAB P300DS (Mo $\mathrm{K}_{\alpha}, \lambda=0.71073 \AA$ ) at $230 \mathrm{~K}$ and $450 \mathrm{~K}$ for the ferroelectric phase and paraelectric phase, respectively. The Crystalclear software package (Rigaku) was used for data collection, cell refinement and data reduction. Using the Olex2 program, the structures were solved by using Intrinsic Phasing with the SHELXT structure solution program and full-matrix least-squares method with the SHELXL refinement program. ${ }^{52,53}$ HKL 5 refinement was applied for the twinning structure of the ferroelectric phase. Non-hydrogen atoms were refined anisotropically and the positions of the hydrogen atoms were generated geometrically. Crystallographic data and structural refinements are summarized in Table S1 (ESI $\dagger$ ). Powder X-ray diffraction (PXRD) patterns ( $\mathrm{Cu} \mathrm{K}_{\alpha}$, and $\lambda=1.54184 \AA$ ) were collected on a Bruker Advance D8 DA VANCI $\theta-2 \theta$ diffractometer.

\section{Materials and physical property measurements}

Elemental (C, H, and N) analyses were performed on a PerkinElmer Vario EL elemental analyzer with as-synthesized samples. Thermogravimetric analysis was carried out using a TA Q50 system with a heating rate of $10 \mathrm{~K} \mathrm{~min}^{-1}$ under a nitrogen atmosphere. Differential scanning calorimetry was done with a TA DSC Q2000 instrument in a nitrogen atmosphere in aluminum crucibles with heating and cooling rates of $10 \mathrm{~K} \mathrm{~min}^{-1}$ from 300 to $450 \mathrm{~K}$. The dielectric measurements were conducted on a TH2828A impedance analyzer with an applied voltage of $1.0 \mathrm{~V}$ and a temperature sweeping rate of $3 \mathrm{~K} \mathrm{~min}^{-1}$ in the temperature range of 300-450 K using a MercuryiTC cryogenic environment controller of Oxford Instruments. Variable-temperature second harmonic generation (SHG) experiments were conducted by Kurtz-Perry powder SHG test using a Nd:YAG laser (1064 nm) with the input pulse of $570 \mathrm{~V}$ under a programmable cryogenic cooling system. The value of nonlinear optical coefficient for TMC-4 has been determined by comparison with a potassium dihydrogen phosphate (KDP) reference. A standard scheme of single-beam Michelson homodyne interferometer was used for the measurements of the electric field-induced displacements. Polarization hysteresis loops were recorded on a Radiant Precision Premier II ferroelectric tester. PFM imaging was done using a commercial scanning probe microscopy system (NTEGRA Aura scanning probe microscope NT-MDT, Russia) equipped with the HF2LI lock-in amplifier (Zurich Instruments, Switzerland), and Krohn-Hite wideband amplifier (7602M, USA). PFM scans were rastered with a single frequency $(21.11 \mathrm{kHz})$ far from the contact resonance frequency $(\sim 190 \mathrm{kHz})$. A cantilever having a sharp $\mathrm{Cr} / \mathrm{Pt}$ conductive coated tip (Budget Sensors) with a spring constant of $48 \mathrm{~N} \mathrm{~m}^{-1}$ and resonance frequency of $190 \mathrm{kHz}$ was used.

\section{Results and discussion}

\section{Thermal analysis}

Thermogravimetric analysis showed that TMC-4 decomposes at $\sim 500 \mathrm{~K}$ in a $\mathrm{N}_{2}$ flow (Fig. S2, ESI $\dagger$ ). Thermally-induced phase transition was verified by differential scanning calorimetry (DSC). Two pairs of endothermic/exothermic peaks at $417 /$ $409 \mathrm{~K}\left(T_{1}\right)$ and $427 / 423 \mathrm{~K}\left(T_{2}\right)$ were observed in a heating/ cooling cycle, respectively (Fig. 1a). It should be noted that only a pair of peaks was observed in Ref. 50. This may be due to the low sensitivity of the instrument used. The large entropy change $\Delta S$ around $T_{1}$ was estimated to be $\sim 178.79 \mathrm{~J} \mathrm{~K}^{-1} \mathrm{~kg}^{-1}$, while small $\Delta S$ around $T_{2}$ was $1.21 \mathrm{~J} \mathrm{~K}^{-1} \mathrm{~kg}^{-1}$. After eight DSC heating-cooling cycles, no significant decrease in the entropy changes around $T_{1}$ and $T_{2}$ was observed, proving that TMC-4 has better $\Delta S$ reversibility and thermal stability than its isostructural 
(a)

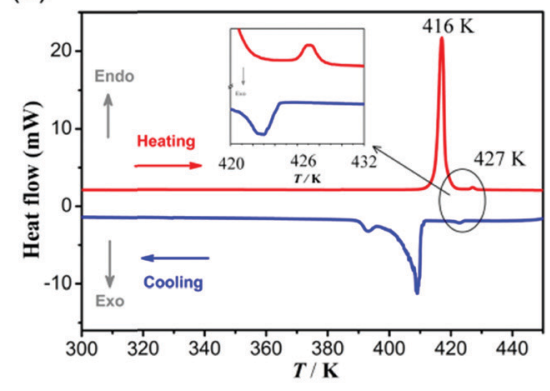

(d)

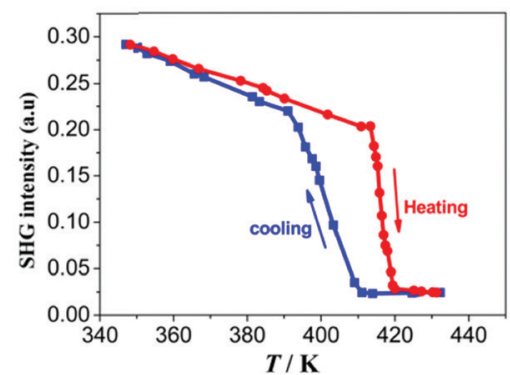

(b)

(e)

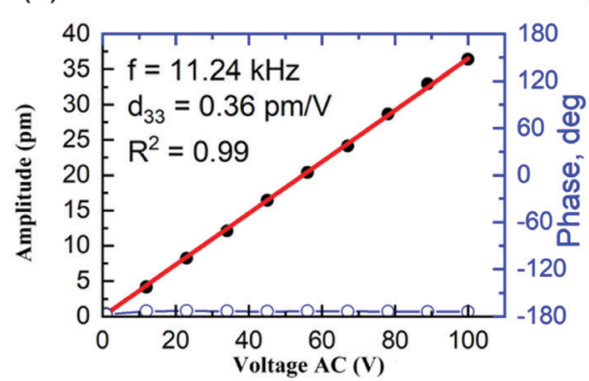

(c) Paraelectric phase $F m \overline{3} m$

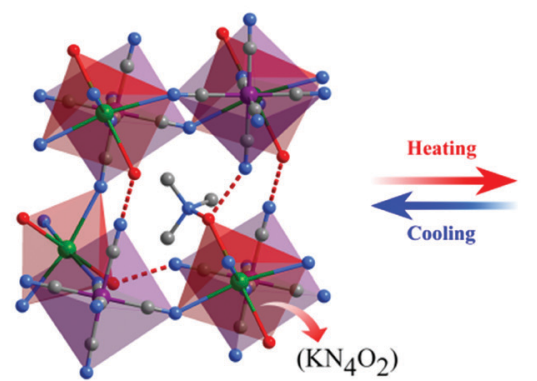

(f)

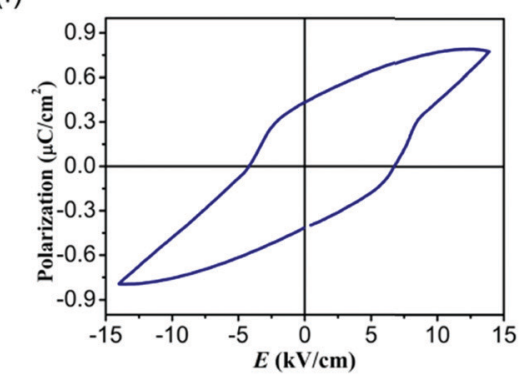

Fig. 1 TMC-4 (a) DSC curves. (b and c) Crystal structures at the ferroelectric and paraelectric phases. (d) SHG signal as a function of temperature. (e) Inverse piezoelectric response. (f) Room-temperature hysteresis loop measured on a single crystal along the ac-plane at $1 \mathrm{~Hz}$.

analogue TMC-1 (Fig. S3, ESI $\dagger$ ), in accordance with the early observations. ${ }^{51}$ The entropy change in TMC-4 is larger than that in other hybrids, ${ }^{54}$ such as (TPrA) $\left[\mathrm{Mn}(\mathrm{dca})_{3}\right]\left(42.5 \mathrm{~J} \mathrm{~K}^{-1} \mathrm{~kg}^{-1}\right){ }^{55}$ $\left(\mathrm{Me}_{4} \mathrm{~N}\right)_{2}\left[\mathrm{CrNa}\left(\mathrm{N}_{3}\right)_{6}\right]\left(100.03 \mathrm{~J} \mathrm{~K}^{-1} \mathrm{~kg}^{-1}\right){ }^{56}$ and $\left(\mathrm{Me}_{3} \mathrm{NNH}_{2}\right)_{2}{ }^{-}$ $\left[\mathrm{Co}(\mathrm{CN})_{6} \mathrm{Na}\left(\mathrm{H}_{2} \mathrm{O}\right)\right]\left(146 \mathrm{~J} \mathrm{~K}^{-1} \mathrm{~kg}^{-1}\right) .{ }^{57} \mathrm{TMC}-4$ is, thus, promising as a thermal storage and barocaloric material. ${ }^{58,59}$

\section{Crystal structure}

In situ variable-temperature single-crystal structural analysis revealed that TMC-4 crystallizes in a polar monoclinic space group $C c$ at $230 \mathrm{~K}$, and in a centrosymmetric cubic space group $F m \overline{3} m$ at $450 \mathrm{~K}$. Hence, the ferroelectric phase (FP) appears below $T_{1}$ and the paraelectric phase (PP) above $T_{2}$. The intermediate phase structure, between $T_{1}$ and $T_{2}$, is difficult to evaluate because it is stable in a very narrow temperature range. In $\mathrm{FP}$, four $\mathrm{N}$ atoms of cyano groups and two hydroxyl groups from two $\mathrm{Me}_{3} \mathrm{NOH}^{+}$cations coordinate to $\mathrm{K}^{+}$ions forming an elongated $\mathrm{KN}_{4} \mathrm{O}_{2}$ octahedron. $\left[\mathrm{Co}(\mathrm{CN})_{6}\right]^{3-}$ and $\mathrm{KN}_{4} \mathrm{O}_{2}$ octahedra are linked via four cyano-bridge ligands and two types of $\mathrm{O}-\mathrm{H} \cdots \mathrm{N}$ hydrogen bonds (ONN distances of 2.648(5) and 2.645(5) $\AA$ ), forming a quasi-perovskite structure with $\mathrm{Me}_{3} \mathrm{NOH}^{+}$cations occupying the voids (Fig. 1b). Above $427 \mathrm{~K}\left(T_{2}\right)$, PP shows an ideal three-dimensional double-perovskite structure hosting $\mathrm{Me}_{3} \mathrm{NOH}^{+}$within the cage, thus revealing 24-fold disorder, as required by the cubic crystallographic symmetry (Fig. 1c).

Such unique TMC-4 phase transition comprises the reversible break and reformation of weak $\mathrm{K}-\mathrm{N}$, and $\mathrm{K}-\mathrm{O}$ coordination bonds, and $\mathrm{O}-\mathrm{H} \cdots \mathrm{N}$ hydrogen bonds, and we refer to it as a bondswitching transition, as suggested for the TMC-1 analogue. ${ }^{49}$ Similarly, the symmetry breaking with an Aizu notation $\mathrm{m} \overline{3} \mathrm{mFm}$ during the bond-switching transition enables TMC-4 to be a multiaxial ferroelectric with 24 equivalent polarization directions in the ferroelectric phase. However, unlike TMC-1, the cell volume of TMC-4 shrinks $0.45 \%$ (from $1877.5 \AA^{3}$ at $230 \mathrm{~K}$ to $1869.0 \AA^{3}$ at $450 \mathrm{~K}$ ) during the bond-switching transition, indicating a negative thermal expansion. ${ }^{60-62}$

\section{SHG, dielectric, piezoelectric and basic ferroelectric properties}

The bond-switching ferroelectric phase transition was further evaluated by variable temperature second harmonic generation (SHG). SHG signals gradually decrease upon heating and vanish above $T_{1}$, indicating that FP is indeed a polar phase, and that both the intermediate phase and PP are non-polar (Fig. 1d). Upon repeating the cooling process, in situ measurement of SHG signals shows a good reversibility. Additionally, the dielectric properties of TMC- 4 were measured at the frequency of $1 \mathrm{MHz}$. A single step-like dielectric anomaly was found in the vicinity of $T_{1}$, and no noticeable change was observed in the vicinity of $T_{2}$ upon a heating-cooling cycle (Fig. S4, ESI $\dagger$ ). Considering the dielectric response and small entropy change in the vicinity of $T_{2}$, we speculate that there is no distinct variation of the motional orientation of the polar $\mathrm{Me}_{3} \mathrm{NOH}^{+}$cations between the intermediate phase and PP.

The inverse piezoelectricity for single crystals of TMC-4 was measured with a Michelson-Morley interferometer. As shown in Fig. 1e, the amplitude of the displacement is linear with the applied voltage amplitude of up to $100 \mathrm{~V}$. The effective piezoelectric coefficient $d_{33}$ is estimated to be $0.36 \mathrm{pm} \mathrm{V}^{-1}$, which is smaller than those of recently reported multiaxial molecular ferroelectrics. ${ }^{36,63}$ This is due to the fact that the crystals were not poled and only a small part of the polarization participated in the piezoelectric response. The macroscopic ferroelectric polarization reversal was investigated on a TMC-4 single crystal along the $a c$-plane at room temperature. A typical ferroelectric 
hysteresis loop was observed at $1 \mathrm{~Hz}$, with an electric polarization of $0.68 \mu \mathrm{C} \mathrm{cm}{ }^{-2}$, slightly larger than that of TMC-1.

\section{Ferroelectric domain structure}

The structural transformation of TMC-4 at the ferroelectric phase transition indicates that it is an improper ferroelectric where spontaneous polarization arises as a secondary effect of structural transformation with a unit-cell doubling. Both ferroelectric properties and dielectric anomalies in improper ferroelectrics are different from those in proper ones. ${ }^{64,65}$ In particular, the temperature-dependence of the permittivity in TMC-4 does not obey the Curie-Weiss law (see Fig. S4, ESI $\dagger$ ), which is a feature of an improper ferroelectric. ${ }^{66}$ The improper ferroelectricity of TMC-4 is reflected in its ferroelectric domain structure with PFM as discussed below.
Amplitude and phase of vertical (out-of-plane, VPFM) and lateral (in-plane, LPFM) components of the piezoelectric response provide valuable information on the local values of the piezoelectric coefficients and orientation of the polarization vector. In the following, for simplicity, we use the basis of the cubic paraelectric phase for crystallographic notations in the FP. PFM was carried out on a $\{111\}_{\mathrm{c}}$ surface (i.e., (002) in the monoclinic basis) of annealed TMC-4 single crystals. Domain boundaries could be unambiguously identified in VPFM and LPFM images without obvious correlation with topography, confirming the existence of ferroelectric domains (Fig. 2 and Fig. S8, S9, ESI $\dagger$ ). The VPFM and LPFM images show different domain distributions and varying contrast in amplitude images, indicating the presence of non $-180^{\circ}$ domain walls, that is, boundaries between domains with non-antiparallel polarization vectors. (a)

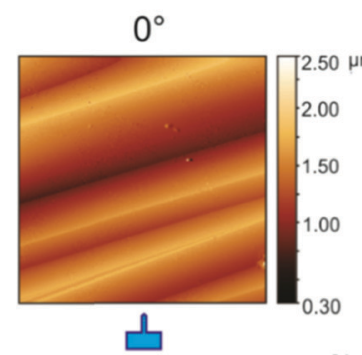

(b)

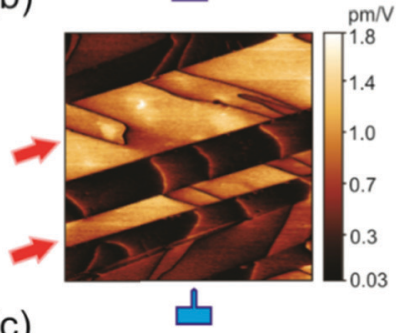

(c)

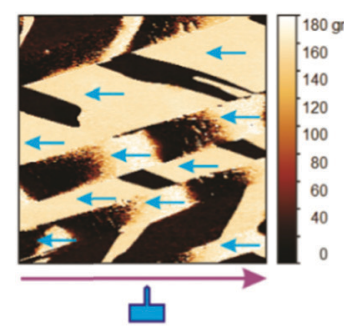

(d)

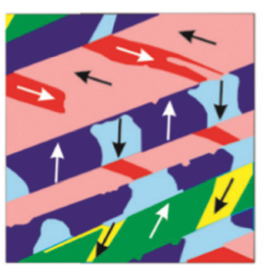

$90^{\circ}$
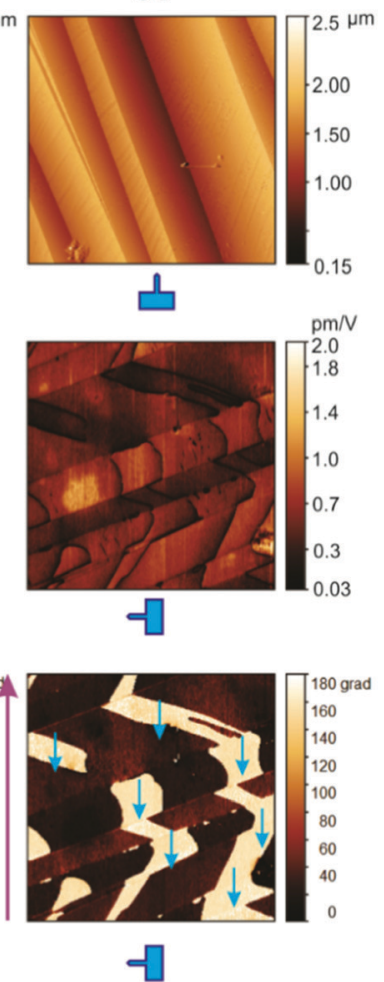

$180^{\circ}$
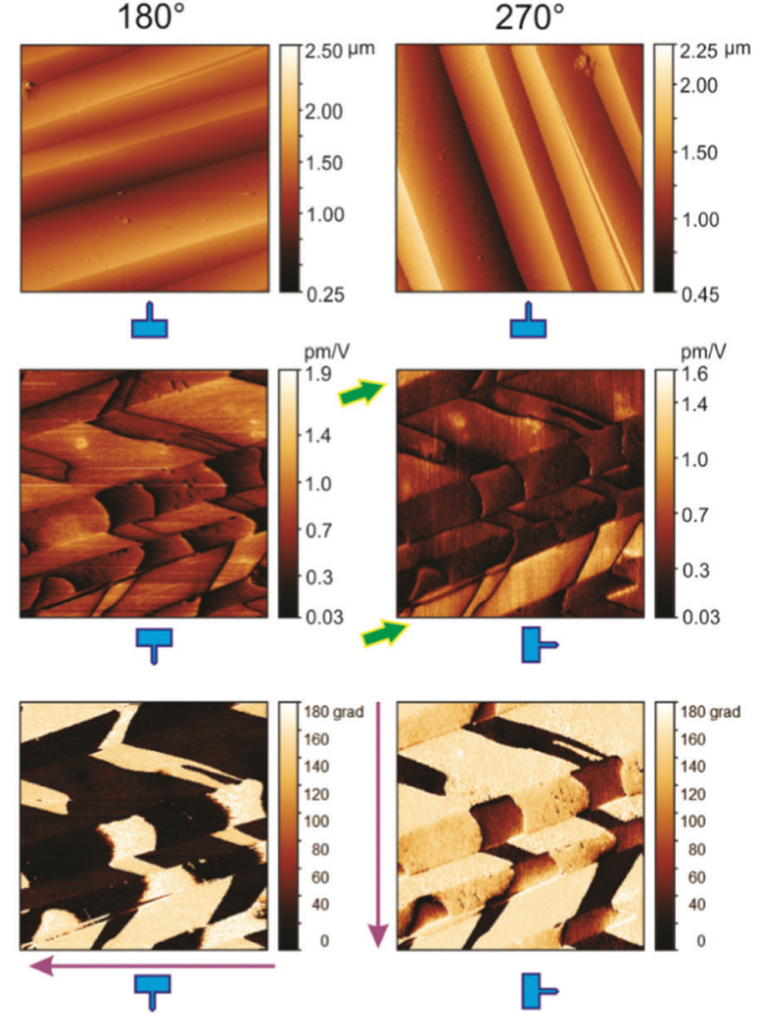

(e)

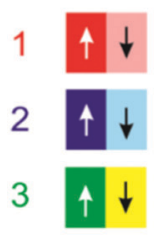

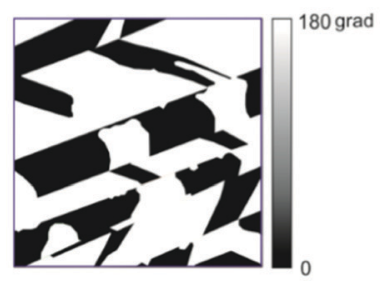

(f)

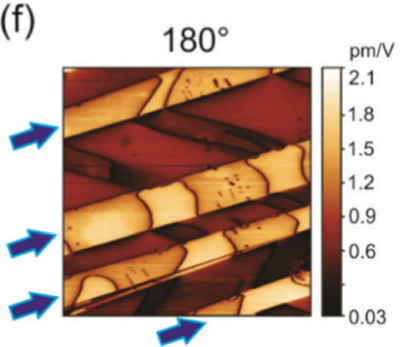

Fig. 2 Maps of (a) topography, (b) LPFM amplitude, and (c) LPFM phase at different angles of the sample rotation around its surface normal. Cantilever orientation relative to images is shown by blue icons under images. Corresponding lateral response directions are shown by dark pink arrows. Blue arrows in (c) indicate directions of projections of lateral polarization in the domains. (d) Distribution of the lateral polarization component deduced from maps in (b) and (c). Maps of (e) VPFM phase and (f) VPFM amplitude at the sample rotation angle of $180^{\circ}$. Red, green, and blue arrows in (b) and (f) indicate bright domain stripes corresponding to red, green, and blue regions in (d), respectively. Image size is $100 \times 100 \mu \mathrm{m}^{2}$. 
To determine polarization directions in the domains, angularresolved LPFM was performed by rotating the sample around its surface normal (Fig. S5, ESI $\dagger$ ). The angular-dependent LPFM images are displayed in Fig. 2 for four angles separated by $90^{\circ}$. Since the LPFM response is determined by the projections of the local polarization vector on the cantilever axis, a map of lateral polarization directions could be obtained and is shown in Fig. 2 d. In the images, we identified three regions with different polarization axes colored in red, blue, and green/yellow in Fig. 2d. Each region consists of $180^{\circ}$ antiparallel ferroelectric domains.

To interpret the observed domain structure, we note that the lattice distortions may be due to the drastic structural phase transition of TMC-4 (see ESI $\dagger$ Fig. S6). If so, the possible ferroelectric domain configurations are imposed by crystal twinning and ferroelastic domain structures (see ESI, $\uparrow$ Fig. S7). The nearly parallel ridges in the topographic images in Fig. 2a are a result of crystal twinning, with ridges and furrows corresponding to ferroelastic domain walls. Each ferroelastic domain (twin) splits into ferroelectric domains separated by $180^{\circ}$ walls. Such walls are electrically neutral and do not have electrostatic energy. In turn, the ferroelastic domain walls coinciding with ferroelectric walls are not necessarily neutral and may have non-compensated positive and negative bound charges. The electrostatic energy of the bound charge is offset by the energy released due to the lattice structure transformation and, therefore, the non- $180^{\circ}$ walls may be a result of the improper nature of the compound's ferroelectricity.

Possible orientations of the ferroelastic domain walls correspond to mirror planes of the parent cubic structure that are lost (a)
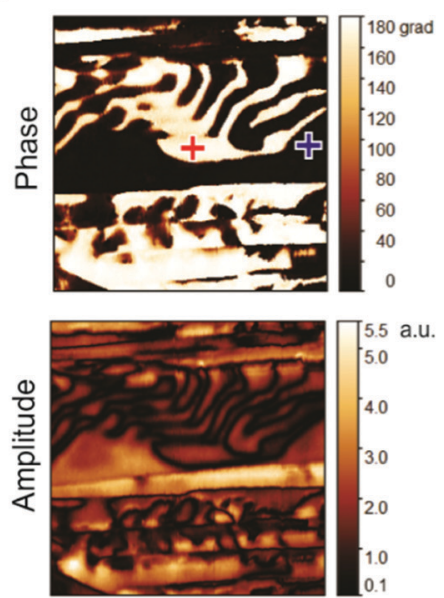

after spectroscopic measurements
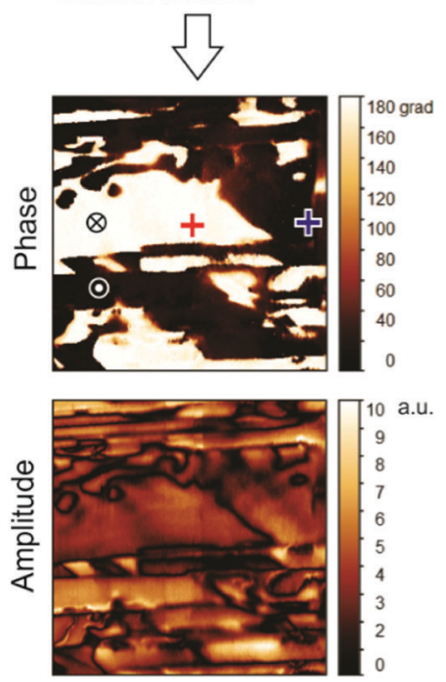

(b)
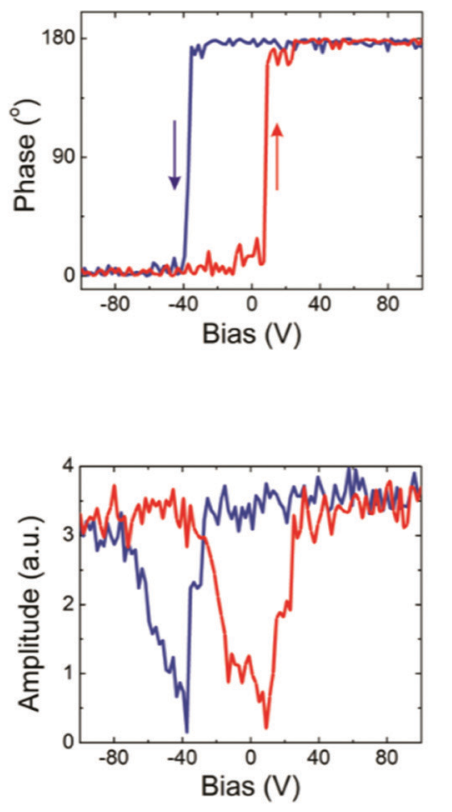

(c)
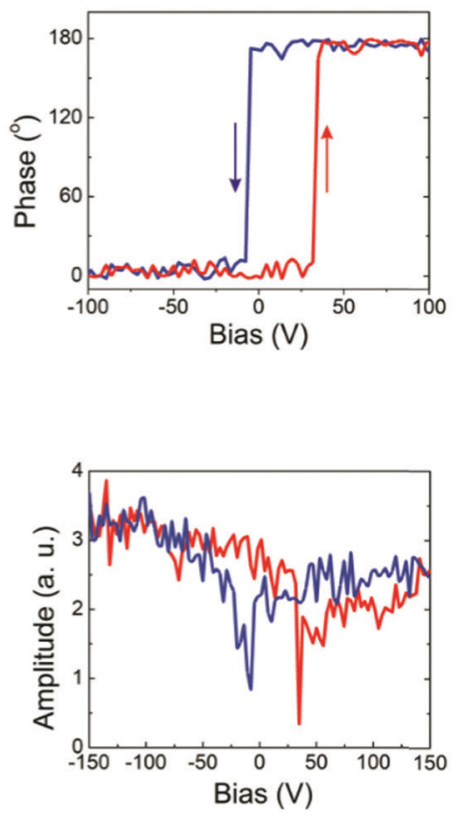

(d)
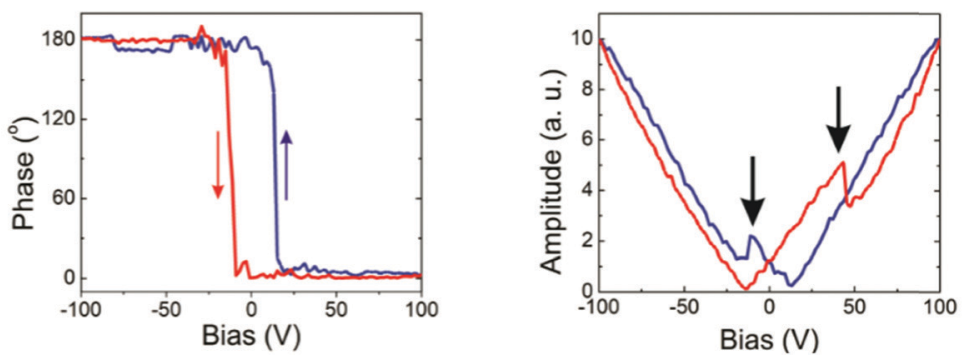

Fig. 3 (a) VPFM phase and amplitude maps before and after spectroscopic measurements. (b) and (c) VPFM spectroscopic measurement loops acquired in the piezo-pulse mode. (d) VPFM spectroscopic measurement loops acquired in the piezo-step mode. The V-shaped electrostatic type loop for amplitude demonstrates ferroelectric switching in the form of two steps that are marked by blue and red arrows, respectively. The VPFM loops in (b) were measured at the center of the area imaged in (a) (marked by red cross) with $U_{\mathrm{ac}}=40 \mathrm{~V}$. The VPFM loops in (c) and (d) were measured at the right side of the area imaged in (a) (marked by blue/white cross) in the piezo-pulse and piezo-step modes with $U_{\mathrm{ac}}=40 \mathrm{~V}$ and $U_{\mathrm{ac}}=20 \mathrm{~V}$, respectively. Image size in (a) is $10 \times 10 \mu \mathrm{m}^{2}$. 
in the ferroelectric phase transition. From the maps in Fig. 2a, there are three types (orientations) of ferroelastic domains. Apparently, these domains are separated by the walls parallel to corresponding mirror planes crossing the surface $(111)_{c}$ plane along straight parallel lines. For a $\{111\}_{c}$ plane (see ESI $\dagger$ Fig. S7b), there is a pair of mirror planes $-\{110\}_{c}$ and $\{100\}_{c}-$ that cross the $\{111\}_{\mathrm{c}}$ plane along parallel lines. These planes make different angles with the $\{111\}_{\mathrm{c}}$ plane $\left(35.3^{\circ}\right.$ and $\left.125.3^{\circ}\right)$, and therefore, corresponding twins may exhibit opposite VPFM phase, compared with the LPFM phase, as seen in the maps in Fig. $2 \mathrm{c}$ and e. In particular, the green region 3 in Fig. 2d shows the opposite VPFM phase behavior in comparison with that of regions 1 and 2 .

For one pair of such planes, there are four mechanically compatible regions (different twins). Typical width of the regions in Fig. 2 is tens of microns, and for our maximal scan size of $100 \times 100 \mu \mathrm{m}^{2}$ (limited by the AFM system), we observe only three out of four regions in one image. The $C_{3}$ rotation symmetry of the $\{111\}_{\mathrm{c}}$ plane assumes three mechanically compatible groups of such domains rotated by $120^{\circ}$ around the surface normal. One of the examples of such twinning structures is shown in Fig. S9 (ESI $\dagger)$. Both LPFM and VPFM images (see ESI $\dagger$ Fig. S8) indicate that polarization directions are oriented at angles between $0^{\circ}$ and $90^{\circ}$ to the sample surface, i.e., domains reveal both measurable components of the ferroelectric polarization. Vertical piezoelectric response up to $2 \mathrm{pm} \mathrm{V}^{-1}$ and lateral one up to $4 \mathrm{pm} \mathrm{V}^{-1}$ were measured on a single domain.

\section{Polarization switching}

PFM switching spectroscopy was performed in both piezo-pulse and piezo-step regimes for the VPFM signal. All measurements were carried out below the fundamental resonance of the cantilever probe at a frequency of $21 \mathrm{kHz}$. The standard time for measurements of one loop was varied from $8 \mathrm{~s}$ to $30 \mathrm{~s}$. Rectangular phase hysteresis loops and butterfly-like amplitude loops were observed (Fig. 3b and c), witnessing the successful switching of ferroelectric domains. Measured at different locations on the sample surface, VPFM loops are asymmetric and demonstrate shifts relative to negative and to positive bias voltages (Fig. 3b-d). The observed asymmetries are related to the initial domain state at the measurement point. As shown in Fig. 3a, after spectroscopic measurements the dominating polarization orientation expands over a larger area. Such behavior as well as the loop asymmetry can be induced by different elastic strain/strain gradients in the measured regions. Domain switching in the strained regions can be unstable and the initial domain pattern quickly reversed between pulses in the piezo-pulse regime. Therefore, we have also performed measurements in the piezo-step regime in which the PFM signal is a sum of electrostatic and piezoelectric contributions. ${ }^{67}$ Ferroelectric switching was clearly observed in amplitude hysteresis loops as two downward steps depicted by black arrows in Fig. 3d.

In Fig. 4, we demonstrate the domain switching. The images were taken before and after application of positive and negative bias voltages. Application of $+70 \mathrm{~V}$ bias to the center of the imaged area leads to the growth of the white domain in the
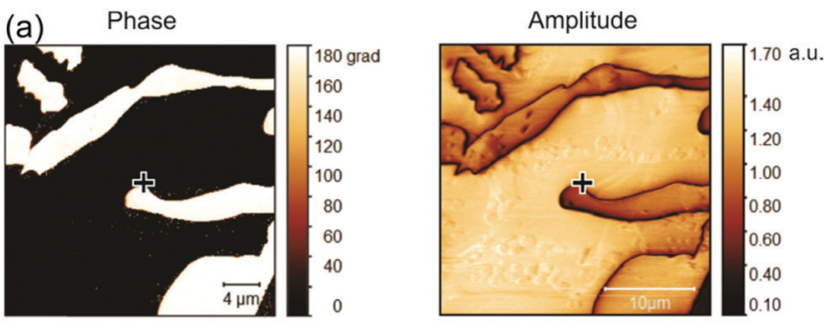

(b)

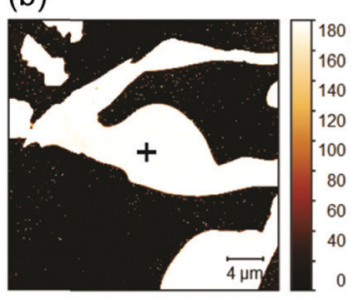

$+70 \mathrm{~V}, 1 \mathrm{~min}$

(c)
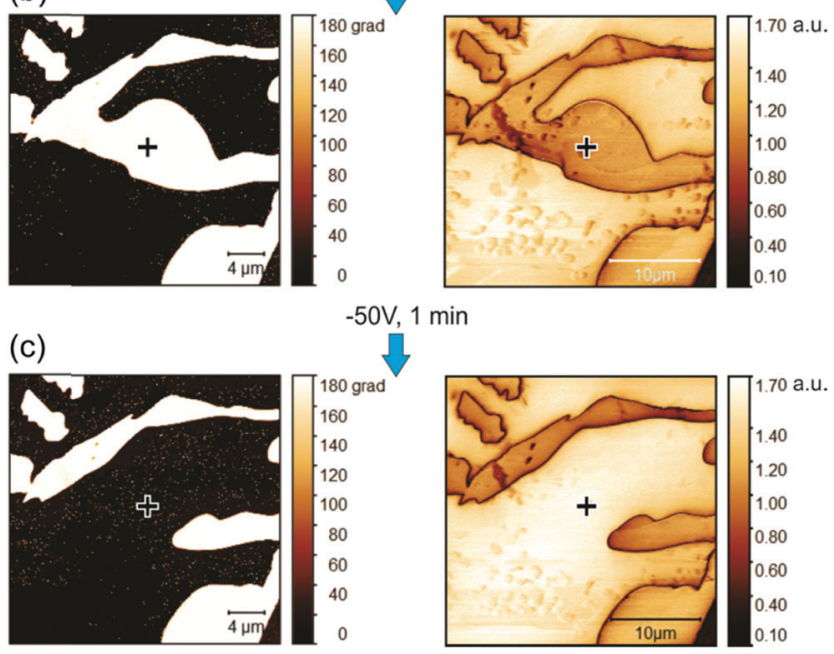

$-50 \mathrm{~V}, 1 \mathrm{~min}$

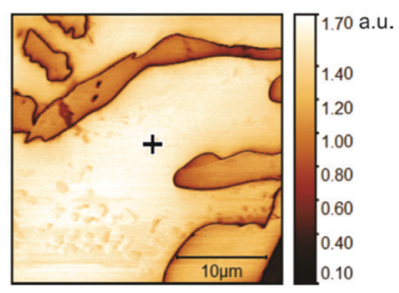

Fig. 4 Domain switching seen in the vertical PFM images after application of a DC pulse at a location in the image center (marked by a black/white cross). Left column: PFM phase; right column: PFM amplitude. Image size is $30 \times 30 \mu \mathrm{m}^{2}$.

vicinity of the point of bias application (Fig. 4b). The following application of $-50 \mathrm{~V}$ leads to a recovery of the initial domain pattern (Fig. $4 \mathrm{c}$ ). The voltage values of $+70 \mathrm{~V}$ and $-50 \mathrm{~V}$ are close to the coercive values measured by the hysteresis loop. Non- $180^{\circ}$ domain switching is suppressed due to the large lattice deformations needed to produce switching between non-antiparallel domain states in neighboring ferroelastic domains. In particular, non- $180^{\circ}$ domain switching requires more time for equilibration and formation of mechanically compatible domain walls. We have demonstrated the permanent non- $180^{\circ}$ domain switching at a bias of $+160 \mathrm{~V}$ during a 10 min-long scan (Fig. S10, ESI $\dagger$ ).

\section{Conclusions}

We have carried out a comprehensive study of TMC-4, a multiaxial perovskite ferroelectric undergoing a bond-switching phase transition at $417 \mathrm{~K}$ accompanied by a large entropy change of 178.79 $\mathrm{J} \mathrm{K}^{-1} \mathrm{~kg}^{-1}$. The material's piezoelectric coefficient $d_{33}$ is about $0.36 \mathrm{pm} \mathrm{V}^{-1}$ in the non-poled sample, and the saturated polarization $0.68 \mu \mathrm{C} \mathrm{cm}^{-2}$. The ferroelectric domain structure of TMC-4 was imaged and found to be determined by the crystal twinning, as expected for an improper ferroelectric. Polarization switching for domains within individual twins was demonstrated. Clear evidence was presented for non- $180^{\circ}$ switching of 
domains between the twins. Furthermore, this work shows that changing the $\mathrm{B}^{\prime \prime}$-site metal ion in this family of double perovskites $\left(\mathrm{Me}_{3} \mathrm{NOH}\right)_{2}\left[\mathrm{~B}^{\prime} \mathrm{B}^{\prime \prime}(\mathrm{CN})_{6}\right]\left(\mathrm{B}^{\prime}=\mathrm{K}^{+}\right.$, and $\mathrm{B}^{\prime \prime}=\mathrm{Fe}^{3+}$, and $\left.\mathrm{Co}^{3+}\right)$ not only tunes their ferroelectricity-related properties (Curie temperature, piezoelectricity, nonlinear optical performance, and spontaneous polarization) but also modifies the thermal properties, such as thermal expansion behaviour, thermal stability, and entropy change. This work prompts further investigation of the origin of the anomalous thermal expansion and barocaloric effects, i.e., isothermal entropy change in the unique TMC-4 multiaxial ferroelectric material. Given that a switchable coordination bond between the $\mathrm{B}^{\prime}$-site and $\mathrm{Me}_{3} \mathrm{NOH}^{+}$is crucial for such a bondswitching transition, changing the $\mathrm{B}^{\prime}$-site provides a strategy for designing novel functional materials based on the bondswitching mechanism.

\section{Conflicts of interest}

There are no conflicts to declare.

\section{Acknowledgements}

This work was supported by the NSFC (22071273, 21805312, and 21821003), and Local Innovative and Research Teams Project of Guangdong Pearl River Talents Program (2017BT01C161). This work (including the grant to W.-J. X.) was also developed within the scope of the project CICECO-Aveiro Institute of Materials, UIDB/50011/2020 \& UIDP/50011/2020, PTDC/CTM-CTM/4044/ 2020 financed by national funds through the FCT/MEC and when appropriate co-financed by FEDER under the PT2020 Partnership Agreement. The equipment of the Ural Center for Shared Use "Modern Nanotechnology" UrFU was used. The work was supported by the Ministry of Science and Higher Education of the Russian Federation (state task FEUZ-2020-0054). It is also funded by National Funds (OE), through FCT-Fundação para a Ciência e a Tecnologia, I.P., in the scope of the framework contract foreseen in the numbers 4, 5 and 6 of the Article 23, of the Decree-Law 57/2016, of August 29, changed by Law 57/2017, of July 19.

\section{Notes and references}

1 D.-W. Fu, H.-L. Cai, Y. Liu, Q. Ye, W. Zhang, Y. Zhang, X.-Y. Chen, G. Giovannetti, M. Capone, J. Li and R.-G. Xiong, Science, 2013, 339, 425-428.

2 Y. Hu, L. You, B. Xu, T. Li, S. A. Morris, Y. Li, Y. Zhang, X. Wang, P. S. Lee, H. J. Fan and J. Wang, Nat. Mater., 2021, 20, 612-617.

3 Q. Pan, Y.-A. Xiong, T.-T. Sha and Y.-M. You, Mater. Chem. Front., 2021, 5, 44-59.

4 S. Horiuchi, Y. Tokunaga, G. Giovannetti, S. Picozzi, H. Itoh, R. Shimano, R. Kumai and Y. Tokura, Nature, 2010, 463, 789-792.

5 Y.-M. You, W.-Q. Liao, D. Zhao, H.-Y. Ye, Y. Zhang, Q. Zhou, X. Niu, J. Wang, P.-F. Li, D.-W. Fu, Z. Wang, S. Gao, K. Yang,
J.-M. Liu, J. Li, Y. Yan and R.-G. Xiong, Science, 2017, 357, 306-309.

6 S. Guerin, A. Stapleton, D. Chovan, R. Mouras, M. Gleeson, C. McKeown, M. R. Noor, C. Silien, F. M. F. Rhen, A. L. Kholkin, N. Liu, T. Soulimane, S. A. M. Tofail and D. Thompson, Nat. Mater., 2018, 17, 180-186.

7 J. Long, M. S. Ivanov, V. A. Khomchenko, E. Mamontova, J.-M. Thibaud, J. Rouquette, M. Beaudhuin, D. Granier, R. A. S. Ferreira, L. D. Carlos, B. Donnadieu, M. S. C. Henriques, J. A. Paixão, Y. Guari and J. Larionova, Science, 2020, 367, 671-676.

8 W. Li, Z. Wang, F. Deschler, S. Gao, R. H. Friend and A. K. Cheetham, Nat. Rev. Mater., 2017, 2, 16099.

9 W.-J. Xu, S. Kopyl, A. Kholkin and J. Rocha, Coord. Chem. Rev., 2019, 387, 398-414.

10 C. Shi, X.-B. Han and W. Zhang, Coord. Chem. Rev., 2019, 378, 561-576.

11 W.-J. Xu, K. Romanyuk, J. M. G. Martinho, Y. Zeng, X.-W. Zhang, A. Ushakov, V. Shur, W.-X. Zhang, X.-M. Chen, A. Kholkin and J. Rocha, J. Am. Chem. Soc., 2020, 142, 16990-16998.

12 S.-S. Wang, X.-X. Chen, B. Huang, R.-K. Huang, W.-X. Zhang and X.-M. Chen, CCS Chem., 2019, 1, 448-454.

13 Z.-S. Yao, K. Yamamoto, H.-L. Cai, K. Takahashi and O. Sato, J. Am. Chem. Soc., 2016, 138, 12005-12008.

14 S. Chen, R. Shang, B.-W. Wang, Z.-M. Wang and S. Gao, Angew. Chem., Int. Ed., 2015, 54, 11093-11096.

15 K. Gao, C. Liu, Z. Cui, C. Xu, J. Zhu, L. Gao, H.-L. Cai and X. S. Wu, J. Mater. Chem. C, 2016, 4, 1959-1963.

16 A. Piecha, A. Gągor, R. Jakubas and P. Szklarz, CrystEngComm, 2013, 15, 940-944.

17 W. Bi, N. Leblanc, N. Mercier, P. Auban-Senzier and C. Pasquier, Chem. Mater., 2009, 21, 4099-4101.

18 M. Ptak, I. E. Collings, K. L. Svane, A. Sieradzki, W. Paraguassu and M. Mączka, J. Mater. Chem. C, 2019, 7, 8660-8668.

19 E. Seyedhosseini, I. Bdikin, M. Ivanov, D. Vasileva, A. Kudryavtsev, B. J. Rodriguez and A. L. Kholkin, J. Appl. Phys., 2015, 118, 072008.

20 H.-Y. Zhang, Y.-Y. Tang, P.-P. Shi and R.-G. Xiong, Acc. Chem. Res., 2019, 52, 1928-1938.

21 H.-Y. Liu, H.-Y. Zhang, X.-G. Chen and R.-G. Xiong, J. Am. Chem. Soc., 2020, 142, 15205-15218.

22 P.-P. Shi, Y.-Y. Tang, P.-F. Li, W.-Q. Liao, Z.-X. Wang, Q. Ye and R.-G. Xiong, Chem. Soc. Rev., 2016, 45, 3811-3827.

23 H. L. B. Boström, M. S. Senn and A. L. Goodwin, Nat. Commun., 2018, 9, 2380.

24 X. Liu, S. Wang, P. Long, L. Li, Y. Peng, Z. Xu, S. Han, Z. Sun, M. Hong and J. Luo, Angew. Chem., Int. Ed., 2019, 58, 14504-14508.

25 S. Wang, X. Liu, L. Li, C. Ji, Z. Sun, Z. Wu, M. Hong and J. Luo, J. Am. Chem. Soc., 2019, 141, 7693-7697.

26 Q. Pan, Z.-B. Liu, Y.-Y. Tang, P.-F. Li, R.-W. Ma, R.-Y. Wei, Y. Zhang, Y.-M. You, H.-Y. Ye and R.-G. Xiong, J. Am. Chem. Soc., 2017, 139, 3954-3957.

27 P.-P. Shi, Y.-Y. Tang, P.-F. Li, H.-Y. Ye and R.-G. Xiong, J. Am. Chem. Soc., 2017, 139, 1319-1324. 
28 Y.-Y. Tang, W.-Y. Zhang, P.-F. Li, H.-Y. Ye, Y.-M. You and R.-G. Xiong, J. Am. Chem. Soc., 2016, 138, 15784-15789.

29 Z.-H. Wei, Z.-T. Jiang, X.-X. Zhang, M.-L. Li, Y.-Y. Tang, X.-G. Chen, H. Cai and R.-G. Xiong, J. Am. Chem. Soc., 2020, 142, 1995-2000.

30 B. Wang, D. Ma, H. Zhao, L. Long and L. Zheng, Inorg. Chem., 2019, 58, 13953-13959.

31 D. Li, X.-M. Zhao, H.-X. Zhao, X.-W. Dong, L.-S. Long and L.-S. Zheng, Adv. Mater., 2018, 30, 1803716.

32 H.-Y. Zhang, S.-Q. Lu, X. Chen, R.-G. Xiong and Y.-Y. Tang, Chem. Commun., 2019, 55, 11571-11574.

33 J. Harada, T. Shimojo, H. Oyamaguchi, H. Hasegawa, Y. Takahashi, K. Satomi, Y. Suzuki, J. Kawamata and T. Inabe, Nat. Chem., 2016, 8, 946-952.

34 J. Walker, R. Miranti, S. L. Skjærvø, T. Rojac, T. Grande and M.-A. Einarsrud, J. Mater. Chem. C, 2020, 8, 3206-3216.

35 S. Das, A. Mondal and C. M. Reddy, Chem. Soc. Rev., 2020, 49, 8878-8896.

36 Y.-Y. Tang, P.-F. Li, W.-Q. Liao, P.-P. Shi, Y.-M. You and R.-G. Xiong, J. Am. Chem. Soc., 2018, 140, 8051-8059.

37 H.-Y. Ye, Y.-Y. Tang, P.-F. Li, W.-Q. Liao, J.-X. Gao, X.-N. Hua, H. Cai, P.-P. Shi, Y.-M. You and R.-G. Xiong, Science, 2018, 361, 151-155.

38 W.-Q. Liao, D. Zhao, Y.-Y. Tang, Y. Zhang, P.-F. Li, P.-P. Shi, X.-G. Chen, Y.-M. You and R.-G. Xiong, Science, 2019, 363, 1206-1210.

39 W.-Q. Liao, Y.-Y. Tang, P.-F. Li, Y.-M. You and R.-G. Xiong, J. Am. Chem. Soc., 2017, 139, 18071-18077.

40 J. Harada, Y. Kawamura, Y. Takahashi, Y. Uemura, T. Hasegawa, H. Taniguchi and K. Maruyama, J. Am. Chem. Soc., 2019, 141, 9349-9357.

41 Q. Pan, Z.-B. Liu, H.-Y. Zhang, W.-Y. Zhang, Y.-Y. Tang, Y.-M. You, P.-F. Li, W.-Q. Liao, P.-P. Shi, R.-W. Ma, R.-Y. Wei and R.-G. Xiong, Adv. Mater., 2017, 29, 1700831.

42 O. Kwon, D. Seol, H. Qiao and Y. Kim, Adv. Sci., 2020, 7, 1901391.

43 Y. Heo, P. Sharma, Y. Y. Liu, J. Y. Li and J. Seidel, J. Mater. Chem. C, 2019, 7, 12441-12462.

44 D. A. Bonnell, S. V. Kalinin, A. L. Kholkin and A. Gruverman, MRS Bull., 2011, 34, 648-657.

45 H. Lu, T. Li, S. Poddar, O. Goit, A. Lipatov, A. Sinitskii, S. Ducharme and A. Gruverman, Adv. Mater., 2015, 27, 7832-7838.

46 Y. Ai, Y.-L. Zeng, W.-H. He, X.-Q. Huang and Y.-Y. Tang, J. Am. Chem. Soc., 2020, 142, 13989-13995.
47 Y.-Y. Tang, Y. Xie, Y. Ai, W.-Q. Liao, P.-F. Li, T. Nakamura and R.-G. Xiong, J. Am. Chem. Soc., 2020, 142, 21932-21937.

48 H.-Y. Zhang, X.-J. Song, X.-G. Chen, Z.-X. Zhang, Y.-M. You, Y.-Y. Tang and R.-G. Xiong, J. Am. Chem. Soc., 2020, 142, 4925-4931.

49 W.-J. Xu, P.-F. Li, Y.-Y. Tang, W.-X. Zhang, R.-G. Xiong and X.-M. Chen, J. Am. Chem. Soc., 2017, 139, 6369-6375.

50 M. Rok, A. Ciżman, B. Zarychta, J. K. Zaręba, M. Trzebiatowska, M. Mączka, A. Stroppa, S. Yuan, A. E. Phillips and G. Bator, J. Mater. Chem. C, 2020, 8, 17491-17501.

51 W.-J. Xu, PhD thesis, Sun Yat-Sen University, 2017.

52 G. M. Sheldrick, Acta Crystallogr., 2015, A71, 3-8.

53 O. V. Dolomanov, L. J. Bourhis, R. J. Gildea, J. A. K. Howard and H. Puschmann, J. Appl. Crystallogr., 2009, 42, 339-341.

54 J. M. Bermúdez-García, M. Sánchez-Andújar and M. A. Señarís-Rodríguez, J. Phys. Chem. Lett., 2017, 8, 4419-4423.

55 J. M. Bermúdez-García, M. Sánchez-Andújar, S. CastroGarcía, J. López-Beceiro, R. Artiaga and M. A. SeñarísRodríguez, Nat. Commun., 2017, 8, 15715.

56 Z.-Y. Du, Y.-P. Zhao, C.-T. He, B.-Y. Wang, W. Xue, H.-L. Zhou, J. Bai, B. Huang, W.-X. Zhang and X.-M. Chen, Cryst. Growth Des., 2014, 14, 3903-3909.

57 W.-J. Xu, Y. Zeng, W. Yuan, W.-X. Zhang and X.-M. Chen, Chem. Commun., 2020, 56, 10054-10057.

58 X. Moya and N. D. Mathur, Science, 2020, 370, 797-803.

59 C. R. Raj, S. Suresh, R. R. Bhavsar and V. K. Singh, J. Therm. Anal. Calorim., 2020, 139, 3023-3049.

60 Z.-Y. Yao, Y. Qian, Q. Ren, G.-Q. Zhang, J. Zhang, W.-L. Liu, H.-B. Luo and X.-M. Ren, J. Mater. Chem. C, 2019, 7, 13243-13252.

61 J. Chen, L. Hu, J. Deng and X. Xing, Chem. Soc. Rev., 2015, 44, 3522-3567.

62 Z.-S. Yao, H. Guan, Y. Shiota, C.-T. He, X.-L. Wang, S.-Q. Wu, X. Zheng, S.-Q. Su, K. Yoshizawa, X. Kong, O. Sato and J. Tao, Nat. Commun., 2019, 10, 4805.

63 C. Shi, J.-J. Ma, J.-Y. Jiang, M.-M. Hua, Q. Xu, H. Yu, Y. Zhang and H.-Y. Ye, J. Am. Chem. Soc., 2020, 142, 9634-9641.

64 A. P. Levanyuk and D. G. Sannikov, Phys. -Usp., 1974, 17, 199-214.

65 A. K. Tagantsev, E. L. Cross and J. Fousek, Domains in Ferroic Crystals and Thin Films, Springer, New York, 2010.

66 B. A. Strukov and A. P. Levanyuk, Ferroelectric phenomena in crystals: physical foundations, Springer, Berlin, 1998.

67 S. Hong, J. Woo, H. Shin, J. U. Jeon, Y. E. Pak, E. L. Colla, N. Setter, E. Kim and K. No, J. Appl. Phys., 2000, 89, 1377-1386. 